\title{
EXTENSIONS OF THE HARDY-LITTLEWOOD INEQUALITIES FOR SCHWARZ SYMMETRIZATION
}

\author{
H. HAJAIEJ and C. A. STUART
}

Received 4 February 2004

For a class of functions $H:(0, \infty) \times \mathbb{R}_{+}^{2} \rightarrow \mathbb{R}$, including discontinuous functions of Carathéodory type, we establish that $\int_{\mathbb{R}^{N}} H(|x|, u(x), v(x)) d x \leq \int_{\mathbb{R}^{N}} H\left(|x|, u^{*}(x), v^{*}(x)\right) d x$, where $u^{*}(x)$ and $v^{*}(x)$ denote the Schwarz symmetrizations of nonnegative functions $u$ and $v$.

2000 Mathematics Subject Classification: 26D20, 42C20, 46E30.

1. Introduction. We build on the approach to symmetrization, which we presented in [6], to establish the inequality

$$
\int_{\mathbb{R}^{N}} H(|x|, u(x), v(x)) d x \leq \int_{\mathbb{R}^{N}} H\left(|x|, u^{*}(x), v^{*}(x)\right) d x,
$$

where $u^{*}(x)$ and $v^{*}(x)$ denote the Schwarz symmetrizations of nonnegative functions $u$ and $v$, for a class of functions $H:(0, \infty) \times \mathbb{R}_{+}^{2} \rightarrow \mathbb{R}$ which includes discontinuous functions of Carathéodory type. Apart from its own role in the calculus of variations, this inequality generalizes simultaneously the inequalities

$$
\begin{gathered}
\int_{\mathbb{R}^{N}} F(u(x), v(x)) d x \leq \int_{\mathbb{R}^{N}} F\left(u^{*}(x), v^{*}(x)\right) d x, \\
\int_{\mathbb{R}^{N}} G(|x|, u(x)) d x \leq \int_{\mathbb{R}^{N}} G\left(|x|, u^{*}(x)\right) d x .
\end{gathered}
$$

Inequality (1.2) was first proved by Crowe et al. [2] for continuous functions $F$ that satisfy a condition which we call (CZR) below. In the special case, $F(s, t)=s t$, this condition is satisfied and we obtain the classical Hardy-Littlewood inequality. In this paper, we establish (1.2) and its generalization (1.1) for functions $F$ and $H$ which need not be continuous. Concerning extensions of (1.2), the paper [2] ends with the remark that "any proof involving approximations of $u$ and $v$ by step functions or of $F$ by smooth functions is likely to require some additional hypothesis on $F$." Note that our Corollary 4.7 and Theorem 5.4 are based on approximation of $u$ or $v$ by simple functions, yet they extend (1.2) to functions $F$ that are not necessarily continuous without introducing any additional hypotheses. Moreover, as Remark 5.2 after Corollary 4.7 and Examples 5.7 and 5.8 show, Theorem 5.4 is optimal in the sense that if any of its hypotheses is not satisfied, then we can construct a triple $(F, u, v)$ satisfying the remaining conditions for which (1.2) is false. 
The first proof of the more general inequality (1.1) (for functions defined on a bounded subset of $\mathbb{R}^{N}$ rather than all of $\mathbb{R}^{N}$ ) seems to be due to Tahraoui [8,9] who requires $H$ to be of class $C^{3}$ and who uses rather complicated approximations of $H$ to obtain the result for nonnegative functions $u$ and $v$ in $L^{p}$ under appropriate growth conditions on $H$. More recently, Brock [1] and Draghici [3] have been able to establish (1.1) for continuous functions $H$ which satisfy similar growth conditions without requiring any analog of the condition $\partial_{1} \partial_{2} \partial_{3} H \leq 0$ that was needed by Tahraoui. Motivated by applications in the calculus of variations, our main goal here is to extend (1.1) to cases where $H$ is not necessarily continuous but rather satisfies some conditions of Carathéodory type. Our method requires the assumption that we call (CZR-3) and which corresponds to $\partial_{1} \partial_{2} \partial_{3} H \leq 0$ in the case when $H$ is smooth. In this respect, we obtain a result that is less general than the one due to Brock but it has the advantage of dispensing with his assumption of continuity of $H$ and, furthermore, it establishes (1.1) for a bigger class of functions $u$ and $v$ and it does not require any growth conditions like his on $H$ either.

Our main results are Proposition 4.1, which establishes inequality (1.1) for all symmetrizable functions provided that $H$ satisfies appropriate conditions, and Theorem 4.4 which establishes it for a smaller class of symmetrizable functions under weaker conditions on $H$. As an immediate consequence of Theorem 4.4, we obtain Corollary 4.7 which generalizes the result of [2] to functions $F$ which need not be continuous on $\mathbb{R}_{+}^{2}$. In Theorem 5.4, we adapt our approach in order to extend (1.2) to an even bigger class of functions $F$. Inequalities analogous to (1.1), (1.2), and (1.3) concerning functions defined on a bounded subset of $\mathbb{R}^{N}$ can easily be deduced from the case we deal with here by the procedure we used in [6, Section 6]. Finally we point out that by following closely the proofs of Proposition 4.1 and Theorem 4.4, we can easily find hypotheses under which the inequality

$$
\int_{\mathbb{R}^{N}} H\left(|x|, u_{1}, \ldots, u_{m}\right) d x \leq \int_{\mathbb{R}^{N}} H\left(|x|, u_{1}^{*}, \ldots, u_{m}^{*}\right) d x
$$

is valid. Indeed, (1.4) is established in [5] using different techniques and some applications of this inequality can also be found there.

2. Notation. All statements about measurability refer to the Lebesgue measure $\mu$ on $\mathbb{R}^{N}$ or on $[0, \infty)$, except in Section 5 where we discuss the composition of a Borel measurable function with a Lebesgue measurable function. For $r \geq 0, B(0, r)=\{x \in$ $\left.\mathbb{R}^{N}:|x|<r\right\}$. There is a constant $V_{N}>0$ such that $\mu(B(0, r))=V_{N} r^{N}$ for all $r>0$.

For a measurable subset $A$ of $\mathbb{R}^{N}$ with $\mu(A)<\infty$,

$$
A^{*}=B(0, r) \text {, where } V_{N} r^{N}=\mu(A) \text {. }
$$

Note that $A^{*}$ is open even though $A$ may not be.

The characteristic function of a set $A$ is denoted by $\chi_{A}$.

Let $M_{N}$ denote the set of all extended real-valued functions which are measurable on $\mathbb{R}^{N}$. For $u \in M_{N}$ and $t \in \mathbb{R}$, let

$$
d_{u}(t)=\mu\left(\left\{x \in \mathbb{R}^{N}: u(x)>t\right\}\right)
$$


be its distribution function and set

$$
F_{N}=\left\{u \in M_{N}: 0 \leq u<\infty \text { a.e. on } \mathbb{R}^{N}, d_{u}(t)<\infty \forall t>0\right\},
$$

the set of Schwarz symmetrizable functions. Following the terminology of [6], we say that an element $u \in F_{N}$ is Schwarz symmetric if there exists a nonincreasing function $h:(0, \infty) \rightarrow[0, \infty)$ such that $u(x)=h(|x|)$ for a.e. $x \in \mathbb{R}^{N}$. Its Schwarz symmetrization is denoted by $u^{*}$. As shown in [6, Proposition 2.5], $u, v \in F_{N}$ with $u \leq v$ a.e. on $\mathbb{R}^{N}$ implies that $u^{*} \leq v^{*}$.

Simple functions can be symmetrized in a very explicit way. Let

$$
E_{N}=\left\{u \in F_{N}: u \text { is a simple function }\right\} .
$$

That is, $E_{N}$ is the set of all functions which can be written as

$$
u=\sum_{i=0}^{k} a_{i} \chi_{A_{i}} \quad \text { for some } k \in \mathbb{N},
$$

where $a_{i} \in(0, \infty)$ with $a_{i}>a_{i+1}, A_{i}$ is a measurable subset of $\mathbb{R}^{N}$ with $\mu\left(A_{i}\right)<\infty$ and $A_{i} \cap A_{j}=\varnothing$ for $i \neq j$.

Setting

$$
S_{i}=\cup_{j=0}^{i} A_{j}, \quad S_{i}^{*}=B\left(0, r_{i}\right), \quad \text { for } 0 \leq i \leq k,
$$

it follows that

$$
u^{*}=\sum_{i=0}^{k} a_{i} X c_{i},
$$

where $C_{0}=B\left(0, r_{0}\right)$ and $C_{i}=B\left(0, r_{i}\right) \backslash \overline{B\left(0, r_{i-1}\right)}$ for $i=1, \ldots, k$.

To deal with functions defined on subsets of $\mathbb{R}^{N}$, we use the following conventions. If $\omega$ is a measurable subset of $\mathbb{R}^{N}$, which has finite measure, let $F_{N}(\omega)$ denote the set of all extended real-valued functions $u$ such that

(i) $u$ is measurable on $\omega$,

(ii) $0 \leq u<\infty$ a.e. on $\omega$.

Clearly, $\left.f\right|_{\omega} \in F_{N}(\omega)$ for all $f \in F_{N}$ and, conversely, given any $f \in F_{N}(\omega)$ we have that $\tilde{f} \in F_{N}$ where $\tilde{f}$ is defined by

$$
\tilde{f}= \begin{cases}f(x) & \text { if } x \in \omega, \\ 0 & \text { if } x \in \mathbb{R}^{N} \backslash \omega .\end{cases}
$$

The Schwarz symmetrization of an element $u \in F_{N}(\omega)$ is defined as

$$
u^{*}=\left.\left[(\tilde{u})^{*}\right]\right|_{\omega^{*}}
$$

where $\tilde{u}$ is the extension of $u$ to all of $\mathbb{R}^{N}$ defined in (2.8). By [6, Lemma 6.1(i)],

$$
\widetilde{\left(u^{*}\right)}=(\tilde{u})^{*} \text {. }
$$


In [6], we make frequent use of the following identity which we refer to again below:

$$
\sum_{i=0}^{k} p_{i} q_{i}=\sum_{i=0}^{k} P_{i} Q_{i}
$$

where $P_{i}=p_{i}-p_{i+1}$ for $i=0,1, \ldots, k-1, P_{k}=p_{k}$, and $Q_{i}=\sum_{j=0}^{i} q_{j}$.

3. Preliminaries. In an integral where no domain of integration is indicated, the integration extends over all of $\mathbb{R}^{N}$. A measurable function $f$ is said to be integrable provided that $\int f_{+}(x) d x<\infty$ and $\int f_{-}(x) d x<\infty$, where $f_{+}(x)=\max \{0, f(x)\}$ and $f_{-}(x)=$ $\max \{0,-f(x)\}$, so that $f(x)=f_{+}(x)-f_{-}(x)$ and then $\int f(x) d x=\int f_{+}(x) d x-$ $\int f_{-}(x) d x$. However, $\int f(x) d x$ makes sense even when $f$ is not integrable provided that either $\int f_{+}(x) d x<\infty$ or $\int f_{-}(x) d x<\infty$, in which case $\int f(x) d x$ still stands for $\int f_{+}(x) d x-\int f_{-}(x) d x$.

LEMMA 3.1. Let $f$ and $g$ be measurable functions on $\mathbb{R}^{N}$ such that $\int f_{-}(x) d x<\infty$ and $\int g_{-}(x) d x<\infty$. Then $\int[f+g]_{-}(x) d x<\infty$ and

$$
\int(f+g)(x) d x=\int f(x) d x+\int g(x) d x
$$

Proof. One easily verifies that $[f+g]_{-} \leq f_{-}+g_{-}$, from which it follows that

$$
\int[f+g]_{-}(x) d x \leq \int\left(f_{-}+g_{-}\right)(x) d x=\int f_{-}(x) d x+\int g_{-}(x) d x<\infty .
$$

Furthermore, $[f+g]_{+} \leq f_{+}+g_{+}$and so $\int[f+g]_{+}(x) d x \leq \int f_{+}(x) d x+\int g_{+}(x) d x$. If $\int[f+g]_{+}(x) d x=\infty$, then $\int f_{+}(x) d x+\int g_{+}(x) d x=\infty$ and it follows that

$$
\int(f+g)(x) d x=\int f(x) d x+\int g(x) d x=\infty
$$

in this case.

To deal with the case where $\int[f+g]_{+}(x) d x<\infty$, we observe that

$$
f_{+}=(f+g-g)_{+} \leq[f+g]_{+}+(-g)_{+}=[f+g]_{+}+g_{-},
$$

and, similarly,

$$
g_{+} \leq[f+g]_{+}+f_{-} .
$$

Thus, $\int[f+g]_{+}(x) d x<\infty$ implies that $\int f_{+}(x) d x<\infty$ and $\int g_{+}(x) d x<\infty$. Hence $f$ and $g$ are integrable in this case and the conclusion follows immediately.

The inequalities we deal with involve composite functions. In the calculus of variations, the following definition establishes the standard context for handling the measurability of such compositions. 
Definition 3.2. A function $G:(0, \infty) \times \mathbb{R}_{+} \rightarrow \mathbb{R}$ is called a Carathéodory function when

(1) $G(\cdot, s):(0, \infty) \rightarrow \mathbb{R}$ is measurable on $(0, \infty)$ for all $s \geq 0$,

(2) $G(r, \cdot): \mathbb{R}_{+} \rightarrow \mathbb{R}$ is continuous on $\mathbb{R}_{+}$for all $r \in(0, \infty) \backslash \Gamma$, where $\Gamma$ is a subset of $(0, \infty)$ having one-dimensional measure zero.

An important property of such a function is that the composition $x \mapsto G(|x|, u(x))$ is measurable on $\mathbb{R}^{N}$ for every function $u \in F_{N}$. In the context of inequality (1.1), we introduce the following extension of this notion.

Definition 3.3. A function $H:(0, \infty) \times \mathbb{R}_{+}^{2} \rightarrow \mathbb{R}$ is called a 2-Carathéodory function when

(1) $H(\cdot, s, t)$ is measurable on $(0, \infty)$ for all $s, t \geq 0$,

(2) $H(r, \cdot, t)$ and $H(r, s, \cdot)$ are continuous on $\mathbb{R}_{+}$for all $s, t \geq 0$ and all $r \in(0, \infty) \backslash \Gamma$, where $\Gamma$ is a subset of $(0, \infty)$ having one-dimensional measure zero.

It is easy to check that $x \mapsto H(|x|, u(x), v(x))$ is measurable on $\mathbb{R}^{N}$ for all functions $u, v \in F_{N}$. Indeed, there is a sequence $\left\{u_{k}\right\} \subset E_{N}$ such that $u=\lim _{k \rightarrow \infty} u_{k}$. Since $H(\cdot, s, \cdot):(0, \infty) \times \mathbb{R}_{+} \rightarrow \mathbb{R}$ is a Carathéodory function for all $s \geq 0$, it follows that $H\left(|x|, u_{k}(x), v(x)\right)$ is measurable and hence that $H(|x|, u(x), v(x))=\lim _{k \rightarrow \infty} H(|x|$, $\left.u_{k}(x), v(x)\right)$ is measurable.

The first part of the next definition gives the property introduced by Crowe et al. [2] in their fundamental paper concerning inequality (1.2). In dealing with (1.1) and (1.3) we require properties of a similar nature.

Definition 3.4. A function $F: \mathbb{R}_{+}^{2} \rightarrow \mathbb{R}$ has the property (CZR) when

$$
F(b, d)-F(b, c)-F(a, d)+F(a, c) \geq 0
$$

for all $b \geq a \geq 0$ and $d \geq c \geq 0$.

A function $G:(0, \infty) \times \mathbb{R}_{+} \rightarrow \mathbb{R}$ has the property (CZR-2) when

$$
G(b, d)-G(b, c)-G(a, d)+G(a, c) \leq 0
$$

for all $a, b \in(0, \infty)$ with $b \geq a$ and $d \geq c \geq 0$.

A function $H:(0, \infty) \times \mathbb{R}_{+}^{2} \rightarrow \mathbb{R}$ has the property (CZR-3) when the function $H(\cdot, \cdot, t)-$ $H(\cdot, \cdot, s)$ has the property (CZR-2) for all $t \geq s \geq 0$.

Slight variants of some of our results in [6] concerning inequality (1.3) are useful for our treatment of (1.1) so we present them first.

THEOREM 3.5. Let $G:(0, \infty) \times[0, \infty) \rightarrow \mathbb{R}$ be a Carathéodory function such that

(i) $G_{-}(|x|, 0)$ is integrable on $\mathbb{R}^{N}$,

(ii) $G$ has the property (CZR-2),

(iii) there exists a continuous function $g:[0, \infty) \rightarrow \mathbb{R}$ such that $g(0)=0$ and

$$
\lim _{r \rightarrow \infty}\{G(r, a)-G(r, b)\} \leq g(a)-g(b)
$$

for all $a, b \in[0, \infty)$ with $b \geq a$. 
Then the inequalities

$$
-\infty<\int G(|x|, u(x)) d x \leq \int G\left(|x|, u^{*}(x)\right) d x
$$

hold for all $u \in F_{N}$ such that $g_{-}(u)$ is integrable on $\mathbb{R}^{N}$.

REMARK 3.6. By (ii), for fixed $a, b \in[0, \infty)$ with $b \geq a,\{G(r, a)-G(r, b)\}$ is a nondecreasing function of $r$. Thus the limit in (iii) exists and $\{G(r, a)-G(r, b)\} \leq$ $\lim _{r \rightarrow \infty}\{G(r, a)-G(r, b)\}$ for all $r>0$.

REMARK 3.7. If $\lim _{r \rightarrow \infty} G(r, s)=G^{\infty}(s)$ exists for all $s \geq 0$ and $G^{\infty}:[0, \infty) \rightarrow \mathbb{R}$ is a continuous function, then condition (iii) is satisfied by setting $g=G^{\infty}-G^{\infty}(0)$.

REMARK 3.8. The integrals in the conclusion are well defined since $\int G_{-}(|x|$, $u(x)) d x<\infty$, and $\int G_{-}\left(|x|, u^{*}(x)\right) d x<\infty$. Indeed, putting $a=0$ in (iii), we find that

$$
G(r, 0)-G(r, b) \leq \lim _{r \rightarrow \infty}\{G(r, 0)-G(r, b)\} \leq-g(b)
$$

and so

$$
G_{-}(r, b) \leq G_{-}(r, 0)+g_{-}(b)
$$

for all $r>0$ and $b \geq 0$ since $G_{-}(r, b)=0$ whenever $G_{+}(r, b)>0$. Hence, for any $u \in F_{N}$,

$$
\begin{aligned}
& G_{-}(|x|, u(x)) \leq G_{-}(|x|, 0)+g_{-}(u(x)) \text { for almost all } x \in \mathbb{R}^{N}, \\
& 0 \leq \int G_{-}(|x|, u(x)) d x \leq \int G_{-}(|x|, 0) d x+\int g_{-}(u(x)) d x<\infty
\end{aligned}
$$

provided that $\int g_{-}(u(x)) d x<\infty$. Similarly, $\int G_{-}\left(|x|, u^{*}(x)\right) d x<\infty$.

Proof. By Remark 3.8, we have that $\int G(|x|, u(x)) d x>-\infty$, and, if $\int G(|x|$, $\left.u^{*}(x)\right) d x=\infty$, the conclusion is trivial. Thus we may assume henceforth that $G\left(|\cdot|, u^{*}(\cdot)\right)$ is integrable.

We consider the function $\Phi:(0, \infty) \times[0, \infty) \rightarrow \mathbb{R}$ defined by

$$
\Phi(r, s)=G(r, s)-G(r, 0)-g(s) .
$$

This is a Carathéodory function which satisfies the hypotheses [6, Proposition 5.1(i) and (ii)]. But, for $r \in(0, \infty) \backslash \Gamma$ and $a, b \in[0, \infty)$ with $b \geq a$,

$$
\begin{aligned}
\Phi(r, a)-\Phi(r, b) & =G(r, a)-G(r, b)-\{g(a)-g(b)\} \\
& \leq \lim _{r \rightarrow \infty}\{G(r, a)-G(r, b)\}-\{g(a)-g(b)\} \leq 0,
\end{aligned}
$$

by the monotonicity of $G(r, a)-G(r, b)$ and assumption (iii). Thus we see that $\Phi$ satisfies all the conditions of [6, Proposition 5.1] and so

$$
0 \leq \int \Phi(|x|, u(x)) d x \leq \int \Phi\left(|x|, u^{*}(x)\right) d x \quad \forall u \in F_{N}
$$


that is,

$$
\begin{aligned}
0 & \leq \int G(|x|, u(x))-G(|x|, 0)-g(u(x)) d x \\
& \leq \int G\left(|x|, u^{*}(x)\right)-G(|x|, 0)-g\left(u^{*}(x)\right) d x
\end{aligned}
$$

for all $u \in F_{N}$. But, by [6, Proposition 4.3(ii)],

$$
\int g_{-}(u(x)) d x=\int g_{-}\left(u^{*}(x)\right) d x
$$

for all $u \in F_{N}$ such that $g_{-}(u)$ is integrable and, by hypothesis,

$$
-\infty<\int G_{-}(|x|, 0) d x<\infty
$$

Setting $p(x)=G\left(|x|, u^{*}(x)\right)$ and $q(x)=G(|x|, 0)+g\left(u^{*}(x)\right)$, it follows that $p$ is integrable and $\int q_{-}(x) d x<\infty$. Hence, by (3.17) and Lemma 3.1,

$$
\begin{aligned}
0 & \geq \int q(x)-p(x) d x=\int q(x) d x+\int(-p)(x) d x \\
& =\int q_{+}(x) d x-\int q_{-}(x) d x-\int p(x) d x,
\end{aligned}
$$

from which it follows that $\int q_{+}(x) d x<\infty$ and, consequently, that $q$ is integrable. But now Lemma 3.1 enables us to conclude that

$$
\int G(|x|, u(x))-q(x) d x=\int G(|x|, u(x)) d x-\int q(x) d x
$$

since we know that $\int G_{-}(|x|, u(x)) d x<\infty$. This means that (3.16) and (3.17) can be written as

$$
0 \leq \int G(|x|, u(x)) d x-\int q(x) d x \leq \int p(x) d x-\int q(x) d x
$$

and the conclusion follows.

A variant of (1.3) deals with functions defined on subsets of $\mathbb{R}^{N}$.

Theorem 3.9. Let $G:(0, \infty) \times \mathbb{R}_{+} \rightarrow \mathbb{R}_{+}$be a Carathéodory function and let $\omega$ be a measurable subset of $\mathbb{R}^{N}$ with finite measure. Suppose that

(i) $G(r, \cdot)$ is nondecreasing on $\mathbb{R}_{+}$for all $r \in(0, \infty) \backslash \Gamma$,

(ii) G has the property (CZR-2),

(iii)

$$
\int_{\omega} G(|x|, 0) d x \leq \int_{\omega^{*}} G(|x|, 0) d x
$$


Then

$$
\int_{\omega} G(|x|, u(x)) d x \leq \int_{\omega^{*}} G\left(|x|, u^{*}(x)\right) d x
$$

for all $u \in F_{N}(\omega)$.

Proof. Consider $u \in F_{N}(\omega)$. We may suppose that

$$
\int_{\omega^{*}} G\left(|x|, u^{*}(x)\right) d x<\infty
$$

since otherwise the conclusion is trivial. Using (i) and (iii), it follows that

$$
0 \leq \int_{\omega} G(|x|, 0) d x \leq \int_{\omega^{*}} G(|x|, 0) d x<\infty
$$

Setting $\Phi(r, s)=G(r, s)-G(r, 0)$, we find that $\Phi$ satisfies the hypotheses of [6, Proposition 5.1] and so

$$
0 \leq \int \Phi(|x|, \tilde{u}(x)) d x \leq \int \Phi\left(|x|,(\tilde{u})^{*}(x)\right) d x
$$

using notation (2.8). Thus

$$
0 \leq \int_{\omega} G(|x|, u(x))-G(|x|, 0) d x \leq \int_{\omega^{*}} G\left(|x|, u^{*}(x)\right)-G(|x|, 0) d x
$$

by (2.10) since $\Phi(r, 0)=0$. Using (3.26), it follows that

$$
\begin{aligned}
0 & \leq \int_{\omega} G(|x|, 0) d x \leq \int_{\omega} G(|x|, u(x)) d x \\
& \leq \int_{\omega^{*}} G\left(|x|, u^{*}(x)\right)-G(|x|, 0) d x+\int_{\omega} G(|x|, 0) d x \\
& \leq \int_{\omega^{*}} G\left(|x|, u^{*}(x)\right) d x,
\end{aligned}
$$

completing the proof.

A corollary to the next lemma provides a simple way of ensuring that Theorem 3.9(iii) is satisfied.

LEMMA 3.10. Let $h:(0, \infty) \rightarrow \mathbb{R}_{+}$be a right-continuous function. Then

(i) $h$ is nonincreasing on $(0, \infty)$

if and only if

(ii)

$$
\int_{\omega} h(|x|) d x \leq \int_{\omega^{*}} h(|x|) d x
$$

for all measurable subsets $\omega$ of $\mathbb{R}^{N}$ with finite measure. 
Proof. Suppose that (i) is true and set $L=\lim _{r \rightarrow \infty} h(r)$. Let $u(x)=h(|x|)-L$ and $v(x)=\chi_{\omega}(x)$ for $x \in \mathbb{R}^{N}$. Clearly, $u \in F_{N}$ and $v \in E_{N}$ and so by the Hardy-Littlewood inequality (see, e.g., [6, Proposition 2.3]),

$$
\int u(x) v(x) d x \leq \int u^{*}(x) v^{*}(x) d x,
$$

where

$$
\begin{aligned}
\int u(x) v(x) d x & =\int_{\omega} h(|x|) d x-L \mu(\omega), \\
\int u^{*}(x) v^{*}(x) d x & =\int u(x) \chi_{\omega^{*}}(x) d x=\int_{\omega^{*}} h(|x|) d x-L \mu\left(\omega^{*}\right)
\end{aligned}
$$

since $u=u^{*}$ on $\mathbb{R}^{N}$ by [6, Proposition 2.4(iii)] and $\left(\chi_{\omega}\right)^{*}=\chi_{\omega^{*}}$ by (2.7). But $\mu(\omega)=$ $\mu\left(\omega^{*}\right)$ so it follows that (ii) is satisfied.

Conversely, we suppose that $h$ is not nonincreasing on $(0, \infty)$. We complete the proof by constructing a subset $\omega$ with finite measure such that

$$
\int_{\omega} h(|x|) d x>\int_{\omega^{*}} h(|x|) d x
$$

By our assumption, there exist $P, Q \in(0, \infty)$ with $P<Q$ such that $h(P)<h(Q)$. Using the right-continuity of $h$, there exist $\varepsilon>0$ and $s, t \in(h(P), h(Q))$ such that

$$
h(r) \leq s<t \leq h(R) \quad \forall r \in[P, P+\varepsilon], R \in[Q, Q+\varepsilon]
$$

For any $a \in(P, P+\varepsilon]$, set

$$
\Omega(a)=B(0, P) \cup\left\{x \in \mathbb{R}^{N}: a \leq|x|<Q+\varepsilon\right\} .
$$

Clearly $\Omega(a)$ is a bounded measurable subset of $\mathbb{R}^{N}$ and

$$
\Omega(a)^{*}=B(0, P) \cup\left\{x \in \mathbb{R}^{N}: P \leq|x|<b(a)\right\},
$$

where $b(a)^{N}=P^{N}+(Q+\varepsilon)^{N}-a^{N}$. By choosing $a$ close to $P$, it follows that $b(a) \in$ $[Q, Q+\varepsilon]$ and we consider $\omega=\Omega(a)$ for such a choice of $a$. Then

$$
\begin{aligned}
& \int_{\Omega(a)^{*}} h(|x|) d x-\int_{\Omega(a)} h(|x|) d x \\
& \quad=\int_{P \leq|x|<b(a)} h(|x|) d x-\int_{a \leq|x|<Q+\varepsilon} h(|x|) d x \\
& \quad=\int_{P \leq|x|<a} h(|x|) d x-\int_{b(a) \leq|x|<Q+\varepsilon} h(|x|) d x \\
& \quad \leq s V_{N}\left\{a^{N}-P^{N}\right\}-t V_{N}\left\{(Q+\varepsilon)^{N}-b(a)^{N}\right\} \\
& \quad=(s-t) V_{N}\left\{a^{N}-P^{N}\right\}<0,
\end{aligned}
$$

as required. 
Observing that the proof that (i) implies (ii) makes no use of the right-continuity of $h$, we obtain the following result.

COROLLARY 3.11. If the function $h$ is nonincreasing on $(0, \infty)$, then Lemma 3.10(ii) is satisfied.

4. Main results. We now come to our first result concerning inequality (1.1).

Proposition 4.1. Let $H:(0, \infty) \times \mathbb{R}_{+}^{2} \rightarrow \mathbb{R}$ be a 2-Carathéodory function having the following properties:

(i) $H(r, 0, t)=0$ for all $r>0$ and $t \geq 0$,

(ii) $H(r, \cdot, 0)$ is nondecreasing on $\mathbb{R}_{+}$for all $r>0$,

(iii) $H(r, \cdot, \cdot)$ has the property $(C Z R)$ for all $r>0$,

(iv) $H(\cdot, \cdot, 0)$ has the property $(C Z R-2)$,

(v) $H$ has the property (CZR-3).

Then

$$
0 \leq \int H(|x|, u(x), v(x)) d x \leq \int H\left(|x|, u^{*}(x), v^{*}(x)\right) d x
$$

for all $u, v \in F_{N}$.

Before giving the proof of this result, we make some comments about the hypotheses.

REMARK 4.2. If $H \in C^{3}\left((0, \infty) \times \mathbb{R}_{+}^{2}\right)$, properties (ii)-(v) are equivalent to the following inequalities:

(ii) $\partial_{2} H(r, \cdot, 0) \geq 0$ for all $r>0$,

(iii) $\partial_{2} \partial_{3} H(r, \cdot, \cdot) \geq 0$ for all $r>0$,

(iv) $\partial_{1} \partial_{2} H(\cdot, \cdot, 0) \leq 0$,

(v) $\partial_{1} \partial_{2} \partial_{3} H \leq 0$.

Furthermore, using (ii) and (iii), we see that

$$
\partial_{2} H(r, \cdot, t) \geq \partial_{2} H(r, \cdot, 0) \geq 0 \quad \forall r>0, t \geq 0,
$$

and, by (iv) and (v), that

(vi)

$$
\partial_{1} \partial_{2} H(\cdot, \cdot, t) \leq \partial_{1} \partial_{2} H(\cdot, \cdot, 0) \leq 0 \quad \forall t \geq 0 .
$$

Using (i), we also have that

$$
\partial_{1} H(\cdot, 0, \cdot)=\partial_{3} H(\cdot, 0, \cdot)=\partial_{1} \partial_{3} H(\cdot, 0, \cdot) \equiv 0 .
$$

Now using (vi), (iii), and (v), respectively, we find that

$$
\partial_{1} H \leq 0, \quad \partial_{3} H \geq 0, \quad \partial_{1} \partial_{3} H \leq 0 \quad \text { on }(0, \infty) \times \mathbb{R}_{+}^{2},
$$

and $H \geq 0$ since we have already noted that $\partial_{2} H \geq 0$. 
Thus, for smooth functions satisfying (i), the hypotheses (ii)-(v) of Proposition 4.1 are equivalent to

(A) $\partial_{1} H \leq 0, \partial_{2} H \geq 0$, and $\partial_{3} H \geq 0$,

(B) $\partial_{1} \partial_{2} H \leq 0, \partial_{1} \partial_{3} H \leq 0$, and $\partial_{2} \partial_{3} H \geq 0$,

(C) $\partial_{1} \partial_{2} \partial_{3} H \leq 0$.

REMARK 4.3. As we showed at the beginning of the proof, the hypotheses of Proposition 4.1 imply that $H \geq 0$. Furthermore, the hypotheses of Proposition 4.1 also imply that $H$ has the following properties:

(ii) ${ }^{\prime} H(r, \cdot, t)$ is nondecreasing on $\mathbb{R}_{+}$for all $r>0$ and all $t \geq 0$,

(iv) $H(\cdot, \cdot, t)$ has the property (CZR-2) for all $t \geq 0$,

and, in fact, all the other monotonicity properties analogous to the conditions (A), (B), and $(\mathrm{C})$ that we have formulated for smooth functions.

Proof. We show first that $H \geq 0$. From (i) and (ii), it follows that $H(r, s, 0) \geq 0$ for all $r>0$ and $s \geq 0$. Then, using (iii), we find that, for all $r>0$ and $s, t \geq 0$,

$$
0 \leq H(r, s, t)-H(r, s, 0)-H(r, 0, t)+H(r, 0,0)=H(r, s, t)-H(r, s, 0),
$$

so that $H \geq 0$ on $(0, \infty) \times \mathbb{R}_{+}^{2}$.

Consider now $u \in E_{N}$ and $v \in F_{N}$, where in the notation (2.5)-(2.7),

$$
u=\sum_{i=0}^{k} a_{i} \chi_{A_{i}}, \quad u^{*}=\sum_{i=0}^{k} a_{i} \chi_{C_{i}} .
$$

Then, using (i) and (2.11),

$$
\begin{aligned}
\int H(|x|, u(x), v(x)) d x & =\sum_{i=0}^{k} \int H\left(|x|, a_{i}, v(x)\right) \chi_{A_{i}}(x) d x \\
& =\sum_{i=0}^{k} \int G_{i}(|x|, v(x)) \chi_{S_{i}}(x) d x,
\end{aligned}
$$

where $G_{i}(|x|, t)=H\left(|x|, a_{i}, t\right)-H\left(|x|, a_{i+1}, t\right)$ with $a_{k+1}=0$ and $S_{i}=\cup_{j=0}^{i} A_{j}$.

We claim that the functions $G_{i}:(0, \infty) \times \mathbb{R}_{+}^{2} \rightarrow \mathbb{R}$ satisfy the hypotheses of Theorem 3.9. In fact, by (ii), $G_{i}(r, 0) \geq 0$ for all $r>0$ and, by (iii), $G_{i}(r, \cdot)$ is nondecreasing on $\mathbb{R}_{+}$, from which it follows that $G_{i} \geq 0$. Furthermore, $G_{i}$ has the property (CZR-2) by hypothesis (v). Finally, we note that $G_{i}(\cdot, 0)$ is nonincreasing on $(0, \infty)$ by the hypothesis (iv), so Corollary 3.11 ensures that Theorem 3.9(iii) is satisfied for $\omega=S_{i}$. Hence

$$
0 \leq \int_{S_{i}} G_{i}(|x|, v(x)) d x \leq \int_{B_{i}} G_{i}\left(|x|,\left(\left.v\right|_{S_{i}}\right)^{*}(x)\right) d x
$$

since $\left(S_{i}\right)^{*}=B_{i}$ with $B_{i}=B\left(0, r_{i}\right)$ in the notation (2.6). Recalling (2.8), $\widetilde{\left(\left.v\right|_{S_{i}}\right)}=v \chi_{S_{i}} \leq v$ on $\mathbb{R}^{N}$ and so $\left[\widetilde{\left(\left.v\right|_{S_{i}}\right)}\right]^{*} \leq v^{*}$ by [6, Proposition 2.4(iv)]. Hence, by $(2.10),\left(\left.v\right|_{S_{i}}\right)^{*} \leq v^{*}$ on 
$B_{i}$ and, since we have already observed that $G_{i}(r, \cdot)$ is nondecreasing on $\mathbb{R}_{+}$, it follows that

$$
\int_{B_{i}} G_{i}\left(|x|,\left(\left.v\right|_{S_{i}}\right)^{*}(x)\right) d x \leq \int G_{i}\left(|x|, v^{*}(x)\right) \chi_{B_{i}}(x) d x
$$

On the other hand, again using (i) and (2.11),

$$
\begin{aligned}
\int H\left(|x|, u^{*}(x), v^{*}(x)\right) d x & =\sum_{i=0}^{k} \int H\left(|x|, a_{i}, v^{*}(x)\right) \chi_{C_{i}}(x) d x \\
& =\sum_{i=0}^{k} \int G_{i}\left(|x|, v^{*}(x)\right) \chi_{B_{i}}(x) d x .
\end{aligned}
$$

Hence, combining (4.8) to (4.11), we see that (1.1) is satisfied for all $u \in E_{N}$ and $v \in F_{N}$.

To extend the conclusion to all $u \in F_{N}$, we recall that for any $u \in F_{N}$, there is a sequence $\left\{u_{k}\right\} \subset E_{N}$ such that $u_{k} \leq u_{k+1}$ and $u=\lim _{k \rightarrow \infty} u_{k}$. By [6, Proposition 2.4], we have that $u_{k}^{*} \leq u_{k+1}^{*}$ and $u^{*}=\lim _{k \rightarrow \infty} u_{k}^{*}$. Since we have already shown that

$$
0 \leq \int H\left(|x|, u_{k}(x), v(x)\right) d x \leq \int H\left(|x|, u_{k}^{*}(x), v^{*}(x)\right) d x
$$

for all $v \in F_{N}$, the monotone convergence theorem yields the conclusion for all $u, v \in$ $F_{N}$, since $H(r, \cdot, t)$ is nondecreasing on $\mathbb{R}_{+}$for all $r>0$ and all $t \geq 0$. Indeed, by (iii), for $a \geq b \geq 0$ and $t \geq 0$,

$$
H(r, a, t)-H(r, b, t)-H(r, a, 0)+H(r, b, 0) \geq 0
$$

whereas $H(r, a, 0)-H(r, b, 0) \geq 0$ by (ii).

As we have already observed, the hypotheses of Proposition 4.1 impose several monotonicity conditions on the function $H$. As we now show, these hypotheses can be relaxed, although in some cases it may be necessary to restrict the class of functions for which (1.1) holds to achieve this. In this way we obtain conditions on $H$ which seem very natural for dealing with (1.1) in the calculus of variations.

THEOREM 4.4. Let $H:(0, \infty) \times \mathbb{R}_{+}^{2} \rightarrow \mathbb{R}$ be a 2-Carathéodory function having the following properties.

(i) $H(|x|, 0,0)$ is integrable on $\mathbb{R}^{N}$.

(ii) $H(\cdot, 0, \cdot)$ has the property $(C Z R-2)$.

(iii) $H(r, \cdot, \cdot)$ has the property $(C Z R)$ for all $r>0$.

(iv) $H(\cdot, \cdot, 0)$ has the property $(C Z R-2)$.

(v) $H$ has the property (CZR-3).

(vi) There are continuous functions $g_{1}, g_{2}$ on $\mathbb{R}_{+}$such that

$$
g_{1}(s)=\lim _{r \rightarrow \infty} H(r, s, 0), \quad g_{2}(s)=\lim _{r \rightarrow \infty} H(r, 0, s),
$$

for all $s \geq 0$. 
Then

$$
-\infty<\int H(|x|, u(x), v(x)) d x \leq \int H\left(|x|, u^{*}(x), v^{*}(x)\right) d x
$$

for all $u, v \in F_{N}$ such that $\left[g_{1}\right]_{-} \circ u$ and $\left[g_{2}\right]_{-} \circ v$ are integrable on $\mathbb{R}^{N}$.

REMARK 4.5. Note that (i) and (vi) imply that $g_{1}(0)=g_{2}(0)=0$.

REMARK 4.6. We do not claim that the integrals in the conclusion of Theorem 4.4 are finite, but they are well defined in the following sense. By (ii),

$$
H(R, 0, t)-H(R, 0,0)-H(r, 0, t)+H(r, 0,0) \leq 0
$$

for all $t \geq 0$ and $R, r \in(0, \infty)$ with $R>r$. Letting $R \rightarrow \infty$, we find that

$$
g_{2}(t)-g_{2}(0)-H(r, 0, t)+H(r, 0,0) \leq 0
$$

and hence that

$$
0 \leq H_{-}(r, 0, t) \leq H_{+}(r, 0, t)-g_{2}(t)-H(r, 0,0)
$$

for all $t \geq 0$ and $r \in(0, \infty)$. Since $H_{+}(r, 0, t)=0$ whenever $H_{-}(r, 0, t)>0$, this implies that

$$
0 \leq H_{-}(r, 0, t) \leq\left[g_{2}\right]_{-}(t)+H_{-}(r, 0,0)
$$

for all $t \geq 0$ and $r \in(0, \infty)$. Therefore

$$
0 \leq \int H_{-}(|x|, 0, v(x)) d x \leq \int\left[g_{2}\right]_{-}(v(x))+H_{-}(|x|, 0,0) d x<\infty
$$

for all $v \in F_{N}$ such that $\left[g_{2}\right]_{-} \circ v$ is integrable over $\mathbb{R}^{N}$. Similarly,

$$
0 \leq \int H_{-}(|x|, u(x), 0) d x<\infty
$$

for all $u \in F_{N}$ such that $\left[g_{1}\right]_{-} \circ u$ is integrable over $\mathbb{R}^{N}$.

But, using (iii), we have that

$$
H(r, s, t)-H(r, s, 0)-H(r, 0, t)+H(r, 0,0) \geq 0
$$

for all $s, t \geq 0$ and $r \in(0, \infty)$. Hence

$$
\begin{aligned}
0 & \leq H_{-}(r, s, t) \leq H_{+}(r, s, t)-H(r, s, 0)-H(r, 0, t)+H(r, 0,0) \\
& \leq H_{+}(r, s, t)+H_{-}(r, s, 0)+H_{-}(r, 0, t)+H_{+}(r, 0,0)
\end{aligned}
$$

and so

$$
0 \leq H_{-}(r, s, t) \leq H_{-}(r, s, 0)+H_{-}(r, 0, t)+H_{+}(r, 0,0)
$$


for all $s, t \geq 0$ and $r \in(0, \infty)$ since $H_{+}(r, s, t)=0$ whenever $H_{-}(r, s, t)>0$. Thus

$$
\begin{aligned}
0 & \leq \int H_{-}(|x|, u(x), v(x)) d x \\
& \leq \int H_{-}(|x|, u(x), 0)+H_{-}(|x|, 0, v(x))+H_{+}(|x|, 0,0) d x \\
& <\infty
\end{aligned}
$$

for all $u, v \in F_{N}$ such that $\left[g_{1}\right]_{-} \circ u$ and $\left[g_{2}\right]_{-} \circ v$ are integrable on $\mathbb{R}^{N}$. This means that $\int H(|x|, u(x), v(x)) d x$ is defined unambiguously by

$$
\int H_{+}(|x|, u(x), v(x)) d x-\int H_{-}(|x|, u(x), v(x)) d x
$$

and a similar interpretation applies to $\int H\left(|x|, u^{*}(x), v^{*}(x)\right) d x$.

Proof. In Remark 4.6, we have already shown that both integrals are well defined and that $\int H(|x|, u(x), v(x)) d x>-\infty$ and $\int H\left(|x|, u^{*}(x), v^{*}(x)\right) d x>-\infty$. Thus if $\int H\left(|x|, u^{*}(x), v^{*}(x)\right) d x=\infty$, the conclusion holds and we may assume henceforth that $H\left(|\cdot|, u^{*}(\cdot), v^{*}(\cdot)\right)$ is integrable.

Consider the function $\Phi$ defined by

$$
\Phi(r, s, t)=H(r, s, t)-H(r, s, 0)-H(r, 0, t)+H(r, 0,0) .
$$

One easily verifies that $\Phi$ satisfies the hypotheses of Proposition 4.1. Hence we have that

$$
\begin{gathered}
0 \leq \int H(|x|, u(x), v(x))-H(|x|, u(x), 0)-H(|x|, 0, v(x))+H(|x|, 0,0) d x \\
\leq \int H\left(|x|, u^{*}(x), v^{*}(x)\right)-H\left(|x|, u^{*}(x), 0\right)-H\left(|x|, 0, v^{*}(x)\right)+H(|x|, 0,0) d x \\
\forall u, v \in F_{N} .
\end{gathered}
$$

Next we observe that the function $g(r, s)=H(r, s, 0)$ satisfies the hypotheses of Theorem 3.5 with $g=g_{1}$. Note that (i) implies that $g_{1}(0)=0$. Thus we obtain

$$
-\infty<\int H(|x|, u(x), 0) d x \leq \int H\left(|x|, u^{*}(x), 0\right) d x
$$

for all $u \in F_{N}$ such that $\left[g_{1}\right]_{-} \circ u$ is integrable on $\mathbb{R}^{N}$. A similar argument shows that

$$
-\infty<\int H(|x|, 0, v(x)) d x \leq \int H\left(|x|, 0, v^{*}(x)\right) d x
$$

for all $v \in F_{N}$ such that $\left[g_{2}\right]_{-} \circ v$ is integrable on $\mathbb{R}^{N}$. 
Setting $p(x)=H\left(|x|, u^{*}(x), v^{*}(x)\right)+H(|x|, 0,0)$ and $q(x)=H\left(|x|, u^{*}(x), 0\right)+$ $H\left(|x|, 0, v^{*}(x)\right)$, it follows from Lemma 3.1, (4.29), and (4.30) that $\int q_{-}(x) d x<\infty$. Since $p$ is integrable, Lemma 3.1 and (4.28) imply that

$$
0 \geq \int(q-p)(x) d x=\int q(x) d x+\int(-p)(x) d x=\int q(x) d x-\int p(x) d x
$$

from which it follows that $\int q(x) d x<\infty$.

Setting $Q(x)=H(|x|, u(x), 0)+H(|x|, 0, v(x))$, it follows from (4.29), (4.30), and Lemma 3.1 that $\int Q_{-}(x) d x<\infty$ and

$$
\int Q(x) d x=\int H(|x|, u(x), 0) d x+\int H(|x|, 0, v(x)) d x
$$

Similarly,

$$
\int q(x) d x=\int H\left(|x|, u^{*}(x), 0\right) d x+\int H\left(|x|, 0, v^{*}(x)\right) d x
$$

and (4.29) and (4.30) now show that $\int Q(x) d x \leq \int q(x) d x$. Thus $Q$ is integrable and, setting $P(x)=H(|x|, u(x), v(x))+H(|x|, 0,0)$, Lemma 3.1 yields

$$
0 \leq \int(P-Q)(x) d x=\int P(x) d x+\int(-Q)(x) d x=\int P(x) d x-\int Q(x) d x .
$$

Hence

$$
\begin{aligned}
\int P(x) d x & =\int(P-Q)(x) d x+\int Q(x) d x \\
& \leq \int(P-Q)(x) d x+\int q(x) d x \\
& \leq \int(p-q)(x) d x+\int q(x) d x, \quad(\text { by }(4.28)) \\
& =\int p(x) d x \quad(\text { by }(4.31)) .
\end{aligned}
$$

But Lemma 3.1 shows that

$$
\begin{aligned}
& \int P(x) d x=\int H(|x|, u(x), v(x)) d x+\int H(|x|, 0,0) d x \\
& \int p(x) d x=\int H\left(|x|, u^{*}(x), v^{*}(x)\right) d x+\int H(|x|, 0,0) d x,
\end{aligned}
$$

completing the proof.

We close this section with the observation that Theorem 4.4 already contains a generalization of the result by Crowe et al. [2] concerning inequality (1.2), although further extensions will be obtained in the next section.

COROLLARY 4.7. Let $F: \mathbb{R}_{+}^{2} \rightarrow \mathbb{R}$ be a function such that

(a) $F(s, \cdot)$ and $F(\cdot, s)$ are continuous on $\mathbb{R}_{+}$for all $s \geq 0$,

(b) $F(0,0)=0$,

(c) $F$ has the property (CZR). 
Then

$$
-\infty<\int F(u(x), v(x)) d x \leq \int F\left(u^{*}(x), v^{*}(x)\right) d x
$$

for all $u, v \in F_{N}$ such that $F_{-}(u(\cdot), 0)$ and $F_{-}(0, v(\cdot))$ are integrable on $\mathbb{R}^{N}$.

REMARK 4.8. In [2, Theorem 3], inequality (1.2) is proved under similar hypotheses except that (a) is replaced by the stronger assumption that $F \in C\left(\mathbb{R}_{+}^{2}\right)$. We point out that it is claimed in [2] that (1.2) holds for all $u, v \in F_{N}$ under these assumptions. However, as the following example shows, this clearly requires some qualification and it seems that the integrability of $F(u(\cdot), 0)$ and $F(0, v(\cdot))$ is tacitly assumed in [2, page 437].

EXAMPLE 4.9. Setting $F(s, t)=s-t$, we have that $F \in C\left(\mathbb{R}_{+}^{2}\right)$ and the hypotheses (b) and (c) of Corollary 4.7 are satisfied. Now consider the functions

$$
u(x)= \begin{cases}\frac{1}{1+x} & \text { for } x \geq 0, v(x)=u(-x) \\ 0 & \text { for } x<0, v(x)=u(-x)\end{cases}
$$

Clearly, $u$ and $v \in F_{1}$ with $u^{*}(x)=v^{*}(x)=1 /(1+2|x|)$ for $x \in \mathbb{R}$. Thus $F\left(u^{*}(x)\right.$, $\left.v^{*}(x)\right)=0$ for all $x$, but $\int F_{-}(u(x), v(x)) d x=\int F_{+}(u(x), v(x)) d x=\infty$ so there is no sense in which $\int F(u(x), v(x)) d x$ can be interpreted. Observe that $F_{-}(u(x), 0)=0$ and $F_{-}(0, v(x))=v(x)$. Replacing $F(s, t)=s-t$ by $F(s, t)=t-s$, we see that, if either of the integrals $\int F_{-}(u(x), 0) d x$ or $\int F_{-}(0, v(x)) d x$ is infinite, inequality (1.2) may fail to hold.

5. Borel functions. In this section, we establish inequality (1.2) for a class of functions $F$ which are not even separately continuous.

Definition 5.1. A function $F: \mathbb{R}_{+}^{2} \rightarrow \mathbb{R}$ is called an $H$-Borel function when either

(1) $F(s, \cdot): \mathbb{R}_{+} \rightarrow \mathbb{R}$ is Borel measurable for all $s \geq 0$,

(2) $F(\cdot, s): \mathbb{R}_{+} \rightarrow \mathbb{R}$ is continuous on $\mathbb{R}_{+}$for all $s \geq 0$, or

(3) $F(s, \cdot): \mathbb{R}_{+} \rightarrow \mathbb{R}$ is continuous on $\mathbb{R}_{+}$for all $s \geq 0$,

(4) $F(\cdot, s): \mathbb{R}_{+} \rightarrow \mathbb{R}$ is Borel measurable for all $s \geq 0$.

REMARK 5.2. Whenever $F$ is an $H$-Borel function in what follows, we will assume that properties (1) and (2) are satisfied, since in the case where (3) and (4) hold, we can replace $F$ by $\widetilde{F}(s, t)=F(t, s)$ and recover the former situation.

REMARK 5.3. Suppose that $u, v \in F_{N}$ and that $F: \mathbb{R}_{+}^{2} \rightarrow \mathbb{R}$ is an $H$-Borel function. Then $F(u(\cdot), v(\cdot))$ is Lebesgue measurable on $\mathbb{R}^{N}$. In fact, there is a sequence $\left\{u_{m}\right\} \subset E_{N}$ such that $\lim _{m \rightarrow \infty} u_{m}(x)=u(x)$ for all $x \in \mathbb{R}^{N}$ and consequently $\lim _{m \rightarrow \infty} F\left(u_{m}(x)\right.$, $v(x))=F(u(x), v(x))$. Hence it is enough to show that $F\left(u_{m}(\cdot), v(\cdot)\right)$ is measurable. But, in the notation (2.5),

$$
u_{m}=\sum_{i=0}^{k} a_{i} \chi_{A_{i}}
$$


and so

$$
F\left(u_{m}(x), v(x)\right)=F(0, v(x)) \chi_{Z}(x)+\sum_{i=0}^{k} F\left(a_{i}, v(x)\right) \chi_{A_{i}}(x)
$$

where $Z=\left\{x \in \mathbb{R}^{N}: u_{m}(x)=0\right\}=\mathbb{R}^{N} \backslash \cup_{i=0}^{k} A_{i}$. Since $F\left(a_{i}, \cdot\right)$ is Borel measurable and $v$ is Lebesgue measurable, [7, Theorem 19.B] (with footnote (2) on page 82) shows that $F\left(a_{i}, v(\cdot)\right)$ is Lebesgue measurable on $\mathbb{R}^{N}$ and our assertion follows easily from this.

THEOREM 5.4. Let $F: \mathbb{R}_{+}^{2} \rightarrow \mathbb{R}$ be an $H$-Borel function which satisfies conditions $(b)$ and (c) of Corollary 4.7. Then the inequalities

$$
-\infty<\int F(u(x), v(x)) d x \leq \int F\left(u^{*}(x), v^{*}(x)\right) d x
$$

hold for all $u, v \in F_{N}$ such that $F_{-}(u(\cdot), 0)$ and $F_{-}(0, v(\cdot))$ are integrable on $\mathbb{R}^{N}$.

REMARK 5.5. As in Theorem 4.4, these integrals may not be finite. However, using (b) and (c), we find that, for all $s, t \geq 0$,

$$
F(s, t)-F(s, 0)-F(0, t) \geq 0
$$

and hence that

$$
0 \leq F_{-}(s, t) \leq F_{-}(s, 0)+F_{-}(0, t)
$$

Thus

$$
0 \leq \int F_{-}(u(x), v(x)) d x \leq \int F_{-}(u(x), 0)+F_{-}(0, v(x)) d x<\infty
$$

for all $u, v \in F_{N}$ such that $F(u(\cdot), 0)$ and $F(0, v(\cdot))$ are integrable on $\mathbb{R}^{N}$. Similarly, $\int F_{-}\left(u^{*}(x), v^{*}(x)\right) d x<\infty$.

REMARK 5.6. The following examples show that the conclusion of Theorem 5.4 can fail if either of conditions (b) and (c) of Corollary 4.7 is not satisfied.

EXAMPLE 5.7. Consider the function $F: \mathbb{R}_{+}^{2} \rightarrow \mathbb{R}$ defined by $F(s, t)=\chi_{\{0\}}(t)$. This is an $H$-Borel function that satisfies condition (c) of Corollary 4.7 but not condition (b) since $F(0,0)=1$. Furthermore, $F_{-} \equiv 0$. Let $v: \mathbb{R} \rightarrow[0, \infty)$ be the function defined by

$$
v(x)=e^{-x} \chi_{[0, \infty)}(x) \quad \forall x \in \mathbb{R} .
$$

Clearly $v \in F_{1}$ and $\mu(\{x \in \mathbb{R}: v(x)=0\})=\infty$. However, $v^{*}(x)=e^{-2|x|}$ for all $x \in \mathbb{R}$ and $\mu\left(\left\{x \in \mathbb{R}: v^{*}(x)=0\right\}\right)=0$. Thus, for any $u \in F_{1}$,

$$
\int F(u(x), v(x)) d x=\mu(\{x \in \mathbb{R}: v(x)=0\})=\infty
$$

whereas

$$
\int F\left(u^{*}(x), v^{*}(x)\right) d x=\mu\left(\left\{x \in \mathbb{R}: v^{*}(x)=0\right\}\right)=0 .
$$


The following example was inspired by a similar one given by Draghici [3] in the context of polarization inequalities.

EXAMPLE 5.8. Consider an $H$-Borel function $F: \mathbb{R}_{+}^{2} \rightarrow \mathbb{R}$ such that $F(0,0)=0$ and suppose that condition (c) of Corollary 4.7 is not satisfied. Then there exist four numbers $b \geq a \geq 0$ and $d \geq c \geq 0$ such that

$$
F(b, d)-F(b, c)-F(a, d)+F(a, c)<0 .
$$

Let $A$ and $B$ be measurable subsets on $\mathbb{R}^{N}$ with

$$
A \cap B=\varnothing, \quad 0<\mu(A)=\mu(B)=M<\infty .
$$

Using the notation of (2.5), we now define two simple functions $u$ and $v \in E_{N}$ as follows:

$$
u=a_{0} \chi_{A_{0}}+a_{1} \chi_{A_{1}}, \quad v=b_{0} \chi_{B_{0}}+b_{1} \chi_{B_{1}},
$$

where $a_{0}=b, a_{1}=a, b_{0}=d, b_{1}=c, A_{0}=B_{1}=A$, and $A_{1}=B_{0}=B$. By (2.7),

$$
u^{*}=a_{0} \chi_{c_{0}}+a_{1} \chi_{C_{1}}, \quad v^{*}=b_{0} \chi_{C_{0}}+b_{1} \chi_{C_{1}},
$$

where $\mu\left(C_{0}\right)=\mu\left(C_{1}\right)=M$. Then, since $F(0,0)=0$,

$$
\begin{aligned}
\int F(u(x), v(x)) d x & =\int_{A} F(u(x), v(x)) d x+\int_{B} F(u(x), v(x)) d x \\
& =F\left(a_{0}, b_{1}\right) M+F\left(a_{1}, b_{0}\right) M \\
& =\{F(b, c)+F(a, d)\} M \\
\int F\left(u^{*}(x), v^{*}(x)\right) d x & =\int_{C_{0}} F(u(x), v(x)) d x+\int_{C_{1}} F(u(x), v(x)) d x \\
& =F\left(a_{0}, b_{0}\right) M+F\left(a_{1}, b_{1}\right) M \\
& =\{F(b, d)+F(a, c)\} M
\end{aligned}
$$

so that

$$
\begin{aligned}
& \int F\left(u^{*}(x), v^{*}(x)\right) d x-\int F(u(x), v(x)) d x \\
& \quad=\{F(b, d)+F(a, c)-F(b, c)-F(a, d)\} M<0 .
\end{aligned}
$$

Proof. We set $\widetilde{F}(s, t)=F(s, t)-F(0, t)-F(s, 0)+F(0,0)$ and begin by considering $u \in E_{N}$ and $v \in F_{N}$. Clearly, $\widetilde{F}(0, t) \equiv 0$ and it follows from (c) that $\widetilde{F} \geq 0$. Using the notation (2.5)-(2.7),

$$
\begin{aligned}
\int \tilde{F}(u(x), v(x)) d x & =\sum_{i=0}^{k} \int \tilde{F}\left(a_{i}, v(x)\right) \chi_{A_{i}}(x) d x \\
& =\sum_{i=0}^{k} \int G_{i}(v(x)) \chi_{S_{i}}(x) d x,
\end{aligned}
$$


where we have used (2.11) with

$$
G_{i}(t)=\tilde{F}\left(a_{i}, t\right)-\tilde{F}\left(a_{i+1}, t\right)=F\left(a_{i}, t\right)-F\left(a_{i}, 0\right)-F\left(a_{i+1}, t\right)+F\left(a_{i+1}, 0\right)
$$

and $a_{k+1}=0$.

Clearly $G_{i}: \mathbb{R}_{+} \rightarrow \mathbb{R}$ is a Borel measurable function with $G_{i}(0)=0$ and it follows from (CZR) that $G_{i}$ is nondecreasing on $\mathbb{R}_{+}$. Thus $\left[G_{i}\right]_{-} \equiv 0$, so by [6, Theorem 6.2],

$$
0 \leq \int_{S_{i}} G_{i}\left(\left.v\right|_{S_{i}}(x)\right) d x=\int_{\left(S_{i}\right) *} G_{i}\left(\left(\left.v\right|_{S_{i}}\right)^{*}(x)\right) d x
$$

But $\left(\left.v\right|_{S_{i}}\right)^{*}=\left(v \chi_{S_{i}}\right)^{*}$ on $\left(S_{i}\right)^{*}$ by (2.9) and, by [6, Proposition 2.4(iv)], $\left(v \chi_{S_{i}}\right)^{*} \leq v^{*}$ on $\mathbb{R}^{N}$. The monotonicity of $G_{i}$ implies that

$$
G_{i}\left(\left(\left.v\right|_{S_{i}}\right)^{*}\right) \leq G_{i}\left(v^{*}\right) \quad \text { on }\left(S_{i}\right)^{*}=B_{i}
$$

This shows that

$$
0 \leq \int \widetilde{F}(u(x), v(x)) d x \leq \sum_{i=0}^{k} \int G_{i}\left(v^{*}(x)\right) \chi_{B_{i}}(x) d x .
$$

On the other hand, still using the notation (2.5)-(2.7) and then (2.11),

$$
\begin{aligned}
\int \widetilde{F}\left(u^{*}(x), v^{*}(x)\right) d x & =\sum_{i=0}^{k} \int \widetilde{F}\left(a_{i}, v^{*}(x)\right) \chi_{C_{i}}(x) d x \\
& =\sum_{i=0}^{k} \int G_{i}\left(v^{*}(x)\right) \chi_{B_{i}}(x) d x .
\end{aligned}
$$

Thus

$$
0 \leq \int \tilde{F}(u(x), v(x)) d x \leq \int \tilde{F}\left(u^{*}(x), v^{*}(x)\right) d x
$$

for all $u \in E_{N}$ and $v \in F_{N}$. It follows from the property (CZR) of $F$ that $\widetilde{F}(\cdot, t)$ is nondecreasing on $\mathbb{R}_{+}$for all $t \geq 0$. As in the proof of Proposition 4.1, this means that [6, Proposition 2.4(v)] and the monotone convergence theorem can be used to extend inequality (5.22) to all $u, v \in F_{N}$. Thus

$$
\begin{aligned}
0 & \leq \int F(u(x), v(x))-F(0, v(x))-F(u(x), 0) d x \\
& \leq \int F\left(u^{*}(x), v^{*}(x)\right)-F\left(0, v^{*}(x)\right)-F\left(u^{*}(x), 0\right) d x
\end{aligned}
$$


since $F(0,0)=0$. Using [6, Proposition 4.3(ii)], we find that

$$
\begin{aligned}
& 0 \leq \int F_{-}(0, v(x)) d x=\int F_{-}\left(0, v^{*}(x)\right) d x<\infty, \\
& 0 \leq \int F_{-}(u(x), 0) d x=\int F_{-}\left(u^{*}(x), 0\right) d x<\infty
\end{aligned}
$$

for $u, v \in F_{N}$ such that $F_{-}(u(\cdot), 0)$ and $F_{-}(0, v(\cdot))$ are integrable on $\mathbb{R}^{N}$.

By Remark 5.5 preceding the proof, we know that for all such functions $u$ and $v$,

$$
\int F_{-}(u(x), v(x)) d x<\infty, \quad \int F_{-}\left(u^{*}(x), v^{*}(x)\right) d x<\infty .
$$

Thus $\int F(u(x), v(x)) d x>-\infty$ and, if $\int F_{+}\left(u^{*}(x), v^{*}(x)\right) d x=\infty$, the conclusion holds without further discussion. Hence we assume from now on that $\int F_{+}\left(u^{*}(x), v^{*}(x)\right) d x$ $<\infty$.

Setting $p(x)=F\left(u^{*}(x), v^{*}(x)\right)$ and $q(x)=F\left(0, v^{*}(x)\right)+F\left(u^{*}(x), 0\right)$, it follows from Lemma 3.1 that

$$
\int(q-p)(x) d x=\int q(x) d x+\int(-p)(x) d x=\int q(x) d x-\int p(x) d x,
$$

and by (5.23),

$$
\int(q-p)(x) d x \leq 0
$$

Hence we see that $\int q(x) d x \leq \int p(x) d x<\infty$ and so $q$ is integrable.

Setting $Q(x)=F(0, v(x))+F(u(x), 0)$, inequalities (5.24) and (5.25), together with Lemma 3.1, show that $\int Q_{-}(x) d x<\infty$ and

$$
\int Q(x) d x=\int F(0, v(x)) d x+\int F(u(x), 0) d x .
$$

Similarly,

$$
\int q(x) d x=\int F\left(0, v^{*}(x)\right) d x+\int F\left(u^{*}(x), 0\right) d x,
$$

so that (5.23) yields

$$
\int Q(x) d x \leq \int q(x) d x .
$$

Therefore $Q$ is integrable and Lemma 3.1 now shows that

$$
\begin{aligned}
\int Q(x)-F(u(x), v(x)) d x & =\int Q(x) d x+\int(-F)(u(x), v(x)) d x \\
& =\int Q(x) d x-\int F(u(x), v(x)) d x .
\end{aligned}
$$


Finally,

$$
\begin{aligned}
\int F(u(x), v(x)) d x & =\int F(u(x), v(x))-Q(x) d x+\int Q(x) d x \\
& \leq \int F\left(u^{*}(x), v^{*}(x)\right)-q(x) d x+\int Q(x) d x \quad(\text { by }(5.23)) \\
& \leq \int F\left(u^{*}(x), v^{*}(x)\right)-q(x) d x+\int q(x) d x \quad(\text { by }(5.31)) \\
& \leq \int F\left(u^{*}(x), v^{*}(x)\right) \quad(\text { by }(5.27))
\end{aligned}
$$

and the proof is complete.

EXAMPLE 5.9. Let $h: \mathbb{R}_{+} \rightarrow \mathbb{R}$ be a continuous function and let $k: \mathbb{R}_{+} \rightarrow \mathbb{R}$ be a monotone function. Then $F(s, t)=h(s) k(t)$ is an $H$-Borel function. If $h$ and $k$ are both nondecreasing on $\mathbb{R}_{+}$, it follows that $F$ has the property (CZR). Furthermore, $F(0,0)=0$ provided that $h(0) k(0)=0$. This gives a very simple type of function satisfying the hypotheses of Theorem 5.4 and these functions $F$ are continuous on $\mathbb{R}_{+}^{2}$ only when $k$ is continuous on $\mathbb{R}_{+}$or $h \equiv 0$. Note that examples of this kind do not have the continuity property with respect to rectangles that is discussed in the remarks about extensions of (1.2) in [2, Section 6], unless $k$ is continuous on $\mathbb{R}_{+}$or $h$ is constant. Since, if $R$ is the rectangle in $\mathbb{R}_{+}^{2}$ with corners at $(a, c),(b, c),(b, d)$, and $(a, d)$, then $F(R)=\{h(b)-$ $h(a)\}\{k(d)-k(c)\}$ in the notation of [2].

6. Variants and extensions. First of all we observe that in Definitions 3.2, 3.3, and 5.1 , the assumption of continuity can be replaced by left-continuity without changing the conclusions of our results since our method uses the approximation of a function in $F_{N}$ from below.

Our results concerning inequalities (1.1) and (1.2) are presented in the case of functions defined on all $\mathbb{R}^{N}$. However, it is easy to deduce analogous results for functions defined on subsets of $\mathbb{R}^{N}$ by the procedure which we used in [6, Section 6], so we do not formulate such results here.

Proposition 4.1 requires rather restrictive monotonicity properties of function $H$ but yields inequality (1.1) for all $u, v \in F_{N}$. Our proofs of Theorems 4.4 and 5.4 begin by introducing auxiliary functions $\Phi$ and $\widetilde{F}$ which have additional monotonicity properties not enjoyed by $H$ and $F$. To obtain conclusions concerning $H$ and $F$ from those involving $\Phi$ and $\widetilde{F}$, we impose some integrability assumptions on the functions $u$ and $v$. A variant of this device is to assume that $H$ (or $F$ ) is monotone with respect to one of the variables and to modify its dependence on the other variable. This leads to a result requiring monotonicity of $H$ (or $F$ ) with respect to $u$ and some additional assumption of integrability concerning $v$. Here is one example of what we mean.

THEOREM 6.1. Let $F: \mathbb{R}_{+}^{2} \rightarrow \mathbb{R}$ be an $H$-Borel function which satisfies conditions (b) and (c) of Corollary 4.7 and also

(d) $F(\cdot, t)$ is nondecreasing on $\mathbb{R}_{+}$for all $t \geq 0$. 
Then the inequality

$$
-\infty<\int F(u(x), v(x)) d x \leq \int F\left(u^{*}(x), v^{*}(x)\right) d x
$$

holds for all $u, v \in F_{N}$ such that $F_{-}(0, v(\cdot))$ is integrable on $\mathbb{R}^{N}$.

Proof. Set $\widetilde{F}(s, t)=F(s, t)-F(0, t)$ and then follow the proof of Theorem 5.4.

Finally we mention that there are extensions of (1.1) to inequalities involving more than two functions. Such results have been obtained in [1, 4].

\section{REFERENCES}

[1] F. Brock, A general rearrangement inequality à la Hardy-Littlewood, J. Inequal. Appl. 5 (2000), no. 4, 309-320.

[2] J. A. Crowe, J. A. Zweibel, and P. C. Rosenbloom, Rearrangements of functions, J. Funct. Anal. 66 (1986), no. 3, 432-438.

[3] C. Draghici, Rearrangement inequalities with applications to ratios of heat kernels, preprint, 2003.

[4] H. Hajaiej, Inégalités de symétrisation et applications, Thèse no 2465, EPFL, Lausanne, 2001.

[5]__ Extended Hardy-Littlewood inequalities and some applications, preprint, 2004.

[6] H. Hajaiej and C. A. Stuart, Symmetrization inequalities for composition operators of Carathéodory type, Proc. London Math. Soc. (3) 87 (2003), no. 2, 396-418.

[7] P. R. Halmos, Measure Theory, D. Van Nostrand Company, New York, 1950.

[8] R. Tahraoui, Symmetrization inequalities, Nonlinear Anal. 27 (1996), no. 8, 933-955.

[9] __ Corrigendum: "Symmetrization inequalities", Nonlinear Anal. 39 (2000), no. 4, 535.

H. Hajaiej: Department of Mathematics, University of Virginia, Charlottesville, VA 22902, USA E-mail address: hichem@vi rginia.edu

C. A. Stuart: Institut d'Analyse et Calcul Scientifique (IACS), Faculte des Sciences de Base (FSB), École Polytechnique Fédérale de Lausanne (EPFL), 1015 Lausanne, Switzerland

E-mail address: charles.stuart@epf1.ch 


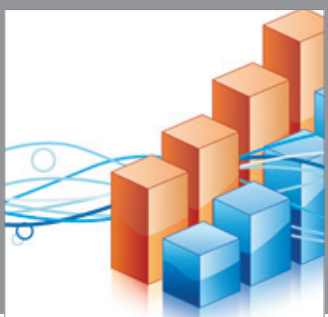

Advances in

Operations Research

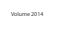

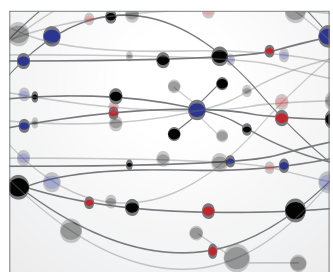

\section{The Scientific} World Journal
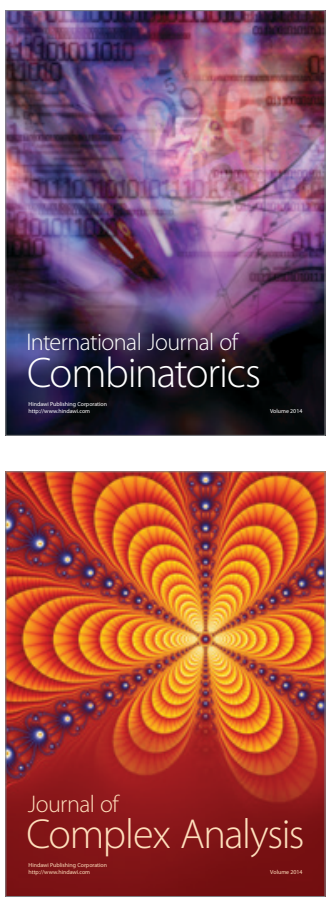

International Journal of

Mathematics and

Mathematical

Sciences
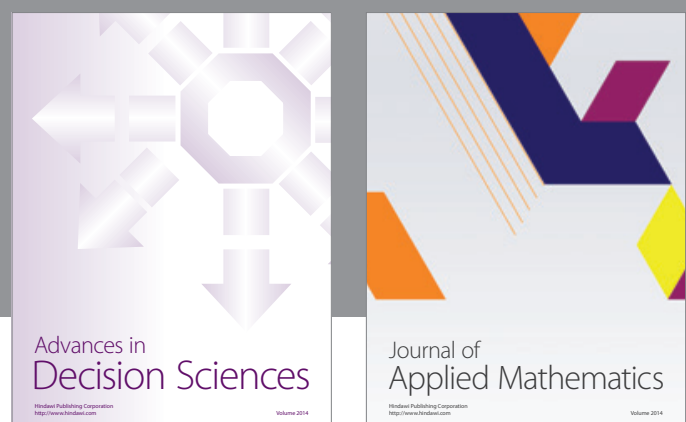

Journal of

Applied Mathematics
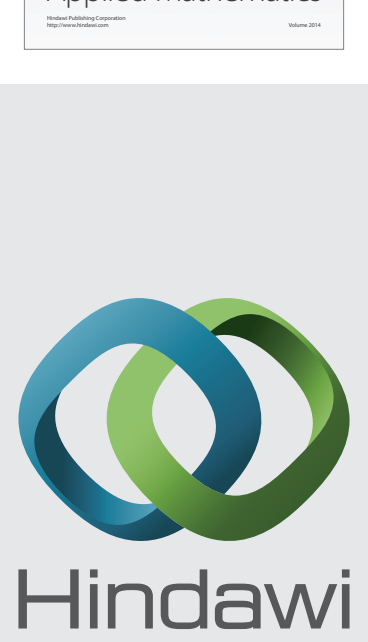

Submit your manuscripts at http://www.hindawi.com
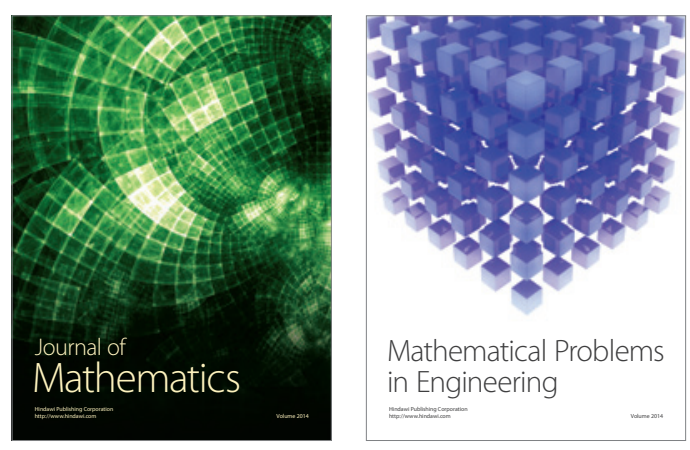

Mathematical Problems in Engineering
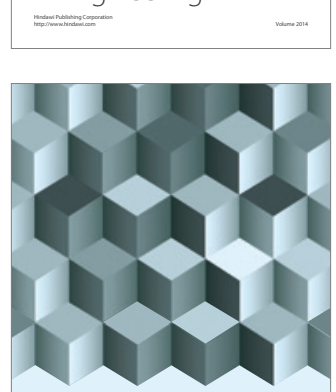

Journal of

Function Spaces
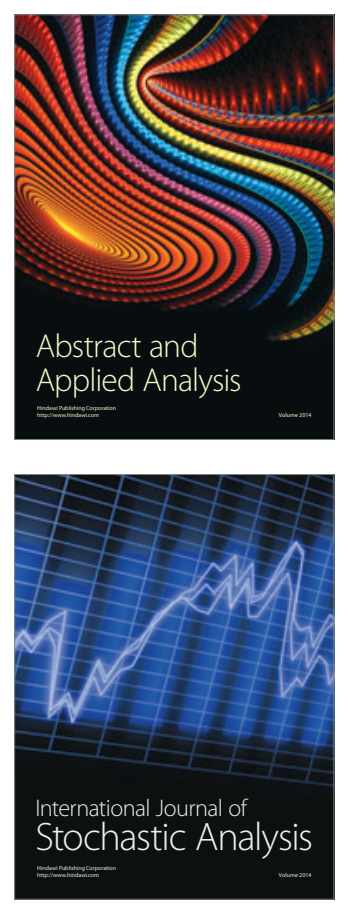

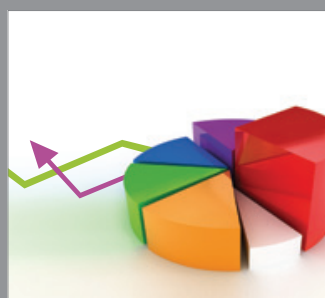

ournal of

Probability and Statistics

Promensencen
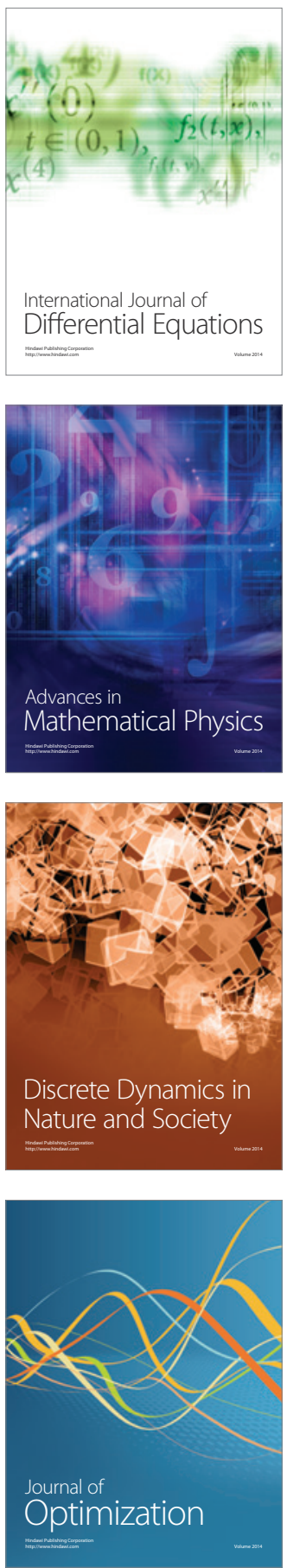